Elsevier required licence: (C) 2018. This manuscript version is made available under the CC-BY-NC-ND 4.0 license http://creativecommons.org/licenses/by-nc-nd/4.0/ 


\title{
Functional relevance of SATB1 in immune regulation and tumorigenesis
}

Krishna P. Sunkara ${ }^{1}$, Gaurav Gupta ${ }^{2}$, Philip M. Hansbro ${ }^{3}$, Kamal Dua $^{1}$, Mary Bebawy ${ }^{1}$

${ }^{1}$ Discipline of Pharmacy, Graduate School of Health, University of Technology Sydney, Ultimo NSW 2007, Australia

${ }^{2}$ School of Pharmaceutical Sciences, Jaipur National University, Jagatpura, 302017, Jaipur, India

${ }^{3}$ School of Biomedical Sciences and Pharmacy, University of Newcastle, Callaghan, NSW 2308, Australia \& Priority Research Centre for Healthy Lungs, Hunter Medical Research Institute, Lot 1 Kookaburra Circuit, New Lambton Heights, Newcastle, NSW 2305, Australia

Correspondence authors:

1. Dr. Krishna Priya Sunkara, Discipline of Pharmacy, Graduate School of Health, University of Technology Sydney, Ultimo NSW 2007, Australia. Email Id: krishna.sunkara@uts.edu.au

2. A/Prof. Mary Bebawy, Discipline of Pharmacy, Graduate School of Health, University of Technology Sydney, Ultimo NSW 2007, Australia. Email Id: mary.bebawy@uts.edu.au

\begin{abstract}
The Special AT-rich Sequence Binding Protein 1 (SATB1) is a chromatin organiser and transcription factor which regulates numerous cellular processes such as differentiation, proliferation and apoptosis through effects on gene expression. SATB1 undergoes various posttranslational modifications, which determine its interaction with co-activators and corepressors to induce regulation of gene transcription. SATB1 is an identified oncogene, its
\end{abstract}


increased expression is associated with poor prognosis in many cancers. This paper provides a review on SATB1-mediated immune responses and on its target genes in the context of tumorigenesis and tumour progression. Specifically, we discuss the role of SATB1 in tumour immunity, Epithelial to Mesenchymal Transition (EMT), metastasis and multidrug resistance. Therapeutic targeting of aberrant SATB1 may be an important strategy in the treatment of cancer.

Keywords: SATB1, tumour, immune responses, gene expression, apoptosis, EMT, invasion, metastasis

\section{Introduction}

The identification of key oncogenic regulators is critical in both defining mechanisms for carcinogenesis as well as for the development of strategies for the diagnosis and treatment of cancer clinically. The special AT-rich sequence-binding protein1 (SATB1) is a nuclear protein and an oncogene, which induces tissue-specific effects on gene regulation and is dysregulated in many cancers [1-3].

SATB1 is a known chromatin organiser and a global regulator of gene expression across various cell types. The genome is anchored to the nuclear matrix through the matrix attachment regions (MARS) [4] . SATB1 specifically binds to the AT-rich motifs of the MAR regions of double stranded DNA [5] and in doing so, it forms a "cage-like" network around heterochromatin, organising it into distinct loops [6-8]. These AT-enriched sites have base unpairing affinity and are known as base unpairing regions (BUR). SATB1 anchored to the BURs provides a docking site for chromatin remodelling proteins and transcription factors for the regulation of many genes [6-9] (Fig 1). Thus, the nuclear organisation of SATB1 tightly controls long-range regulation of genes located distal to the SATB1 bound loci. Post- 
translational modification of SATB1 such as phosphorylation and acetylation are also important in regulating gene expression. These modifications serve as molecular switches, conferring onto SATB1 the ability to act as activator or repressor of gene expression $[10,11]$.

The structure of SATB1 consists of six functional domains including; the nuclear localization signal (NLS) domain, the PDZ domain which facilitates protein interactions, the BUR-binding domain which contains the CUT1 domain and a part of CUT 2 domain, and the homeodomain (HD) which is a DNA binding motif (Fig 2). The BUR and HD domains confer specific and high binding affinity to the core unwinding elements of BURs. Thus through these six domains, SATB1 exerts its function of global gene regulation.

SATB1 functions as gene activator or repressor through its interaction with the chromatin modifying enzymes at the PDZ domain. These interactions are context specific as they are determined by its post-translational modification [10-12]. The phosphorylation of SATB1 by protein kinase C (PKC) at serine186, regulates interaction of SATB1 interaction with histone deacetylase 1 (HDAC1), increasing its DNA binding affinity and facilitating its function as a repressor of gene expression [10, 13]. However, when SATB1 is dephosphorylated, it is acetylated by histone acetyltransferase P300/CBP-associated factor (PCAF), at lysine136. This results in unbinding of SATB1 from DNA, the dissociation of HDAC1 and the de-repression or activation of gene expression [11] (Fig 3).

SATB1 is also regulated by small non-coding RNAs called microRNAs (miRs), which are post-transcriptional regulators of gene expression [14]. The increased expression of miR191 in epidermal keratinocytes induces senescence through the downregulation of SATB1 and Cyclin Dependent Kinase 6 [15]. miR-191 also down regulates SATB1 to promote tumorigenesis in breast cancer [16]. Contrary to this, miR-23a inhibits SATB1 expression in osteosarcoma and acts as a tumour suppressor [17]. SATB1 is also regulated by miR-21, a bona fide onco-miR which is associated with a number cancers including glioblastoma, lung, breast, liver and cervical cancers [18-23]. These studies show that SATB1 is regulated by various 
miRs and the function of SATB1 is both dependent on posttranslational modifications and tissue localisation.

\section{SATB1 and the immune response}

SATB1 is critical for hematopoietic stem cell (HSC) maintenance. HSCs deficient in SATB1 have diminished self-renewal capacity, reduced quiescence and promote myeloid commitment of HSCs [24]. Alternatively, HSCs expressing increased SATB1 levels, differentiate into lymphocyte specific lineage [25]. Importantly, SATB1 regulates T-cell differentiation and is involved in interleukin (IL)-2-mediated T-cell proliferation, differentiation, activation and immune regulation [26]. Consistent with this, SATB1 null mice show impaired T-cell development and are susceptible to death by 3 weeks of age [26]. Likewise, SATB1 conditional knockout mice, show reduced T-cell numbers, poor T-cell differentiation and are susceptible prone to autoimmune disease [27].

SATB1 is required for thymocyte differentiation where it stimulates lineage-specific factors such as T-Helper-Inducing POZ/Krueppel-Like Factor (ThPok), Runt Related Transcription Factor 3 (Runx3), Cluster of differentiation 4 (CD4), CD8 and Forkhead box P3 (FOXP3) [28]. SATB1 binds to $\beta$-catenin in the Wnt signalling pathway, inducing TransActing T-Cell-Specific Transcription Factor (GATA) 3 expression that drives TH2 lineage specification of naïve T-cells [29]. Activation of TH2 cells induces SATB1 which subsequently binds to the TH2- decisive factors GATA3, Signal transducer and activator of transcription 6 (STAT6) and c-Maf to induce TH2 cytokine secretion. Thus, SATB1 induces synchronized expression of TH2 cytokines interleukin (IL)-4, IL-5, IL-13 and c-Maf orchestrating the TH2 cell lineage [8].

SATB1 also plays an important role in mediating immune tolerance. T regulatory cells (Tregs) govern self-tolerance and immune homeostasis. Foxp3 regulates the suppressive 
activity of Tregs through the repression of SATB1. On de-repression of SATB1 from Foxp3, Tregs lose this immunosuppressive capability and the cells differentiate into T-effector (Teff) cells and mediate effector cytokine production [30]. Furthermore, Treg cell-specific superenhancers (Treg-SEs) which are active in Treg precursor cells are dependent on SATB1 and play a vital role in Treg cell commitment [31]. The disruption of SATB1-dependent Treg-SEs activation has been associated with autoimmune and immunodeficiency disorders [31]. These studies support the importance SATB1 in T-cell differentiation and T-cell mediated immune responses.

Contrary to T-cell lineage polarisation, SATB1 does not exert B-cell differentiation, however, it regulates $\mathrm{T}$ - and B-cell lineage specific gene, the recombination-activating gene (Rag) 1, which is important in the differentiation of both these cell types [25]. Besides this, SATB1 deficient lymphoid-primed multipotent progenitors (LMPPs) and common lymphoid progenitor (CLP) lineage cells, show reduced B-cell numbers [25]. This is consistent with the reduced B-cells numbers observed in SATB1 deficient mice [26].

SATB1 also regulates dendritic cell (DC) maturation towards immunocompetent antigen-presenting cells through the expression of major histocompatibility complex (MHC) II [32]. This occurs through the binding of SATB1 to Notch1 homolog, translocation-associated $(\mathrm{NOTCH})$ promoter, stimulating $\mathrm{NOTCH}$ expression and resulting in transcription of major histocompatibility complex (MHC) II [32]. SATB1 interacts with a sub-nuclear structure called promyelocytic leukemia (PML), organizing the MHC class I locus into higher-order chromatin loop domains and regulates the expression of a subset of genes of MHC class I locus [33].

SATB1 governs immune cell development, differentiation and regulates the function of different cell types including the epidermis and neurons [34, 35]. Tumour protein 63 (p63) is a transcription factor and a master regulator of epidermal differentiation [36]. In multipotent progenitor cells, p63 interacts with SATB1 to regulate epidermal morphogenesis [34]. Further, SATB1 is also required for the growth of keratinocytes, its downregulation of which induces 
senescence in neonatal human epidermal keratinocytes [15], indicating its crucial role in cell proliferation and maintenance of the epidermis. SATB1 also plays a vital role in neuronal functioning. In a study on post-natal cortical development, SATB1 was shown to regulate temporal expression of immediate early genes and the formation of dendritic spines that affect the brain function in post-natal neurons [35]. SATB1 was also shown to be a critical regulator of interneuron development and loss of function studies revealed that SATB1 was essential for the differentiation of cortical neurons [37].

\section{SATB1 and Cancer}

Since the discovery of SATB1, its role in the pathogenesis of various cancers has been investigated. Importantly, upregulation of SATB1 has been shown to promote many pathological features across a wide range of cancers [38, 39] including breast [1, 40-42], colorectal [2, 43-45], lung [46, 47], nasopharyngeal [48], oesophageal [3, 49, 50], gastric [51, 52], pancreatic [53, 54], ovarian [32, 55, 56], liver [57-59], prostate [60-63], bladder [64, 65], and brain [66-70]. In most cancers, the SATB1 expression is positively associated with increased tumour size, lymph node involvement and metastasis [71, 72], tumour progression $[1,2]$, poor prognosis $[50,56]$ and reduced overall survival $[49,54]$. SATB1 regulates the expression of more than 1000 genes required for cell-cycle regulation, proliferation, differentiation, adhesion, signalling and apoptosis in breast cancer cells [1], and is an independent prognostic marker across many cancers $[1,56,70,73-76]$. Other studies have reported that SATB1 expression was negatively associated with cancer progression and survival in breast [77] and lung cancers [78]. The basis for these discrepancies and the exact role of SATB1 in cancer pathogenesis remains to be elucidated.

Interestingly, SATB2 a homolog of SATB1 with high sequence homology [79], has been associated with tumour suppressive activity and is of prognostic significance in laryngeal 
squamous cell carcinoma [80] and colorectal carcinoma [81]. In colorectal cancer, SATB2 induces the suppression of extracellular signal-regulated kinase 5 (ERK5) [82], which promotes an aggressive cancer phenotype. In colorectal cancer samples, a negative correlation between SATB1 and SATB2 expression was found, the expression of SATB1 was upregulated and SATB2 was downregulated. SATB1 was shown to upregulate the expression of protooncogene MYC whereas SATB2 downregulated MYC expression [83]. Thus, SATB1 and SATB2 were shown to exert opposite effects on cell proliferation, colony formation and tumour growth $[83,84]$. The role of these closely related genes with an opposing function in tumorigenesis requires further study.

In the following sections, the role of SATB1 in regulating the tumour microenvironment (TME), cell proliferation, apoptosis, invasion, $t$ metastasis and multidrug resistance (MDR) are discussed and are summarised in Fig 4.

\section{SATB1 and the tumour microenvironment}

The tumour microenvironment is a heterogeneous milieu comprising of different cells including cancer cells, stromal cells and the infiltrating immune cells that play an important role in cancer immunity. In a number of tumours, infiltrating CD8+ T-cell numbers were observed to be increased, and this appears to be a favourable clinical outcome in many cancers including early stages of colorectal cancers, breast tumours and ovarian cancers [85-87]. Likewise, in ovarian cancer, a high $\mathrm{CD} 8+$ to Foxp3+ Treg cell ratio within the tumour microenvironment is associated with a positive prognosis $[87,88]$. However, in a study on malignant melanoma, despite a significant presence of activated CD8+ T-cells, tumour progression continued [89]. Importantly the role of Programmed cell death protein-1 (PD-1) is important in evading antitumor immune responses of T-cells [90-92]. The mechanism through which the PD-1 impairs the anti-tumour immune responses of T-cells was recently shown to 
involve SATB1. Normally, activated T-cells were shown to express high SATB1 levels, which recruit a nucleosome remodelling deacetylase complex to repress PDCD1 expression. Consequently, the levels of PD-1 receptor, encoded by PDCD1 are reduced, preventing premature T-cell exhaustion [90]. However, in cancer, the tumour-derived transforming growth factor- $\beta$ (TGF- $\beta$ ) was shown to decrease SATB1 expression in T-cells resulting in de-repressed PDCD1 expression, increased PD-1 levels and inhibited T-cell activity. Thus, altered SATB1 expression potentially impairs anti-tumour immune responses [90].

SATB1 is required for maturation of DCs, but in ovarian cancer, DCs, express a perpetual increase in SATB1 levels, which stimulates secretion of pro-inflammatory mediators and immunosuppressive molecules including IL-6 and galectin, supporting tumour growth [32]. The In vivo knockdown of SATB1 in DCs, reverses the inflammation, tumorigenic activity and increased protective immune responses $[32,93]$. These studies clearly demonstrate that the dysregulation of SATB1 in cancer leads to an altered immune response, which can promote tumorigenesis and tumour progression.

SATB1 was shown to promote the expression of several proto-oncogenes and cell proliferation markers. In glioblastoma cell lines, knockdown of SATB1 led to changes in the expression of various proto-oncogenes such as MYC, B-cell lymphoma 2 (BCL2), Pim-1, epidermal growth factor receptor (EGFR), $\beta$-catenin and Survivin [66]. In pancreatic cancer cells, SATB1 was found to be overexpressed and positively regulated the proto-oncogene MYC [53]. In colorectal carcinoma, increased SATB1 expression was positively associated with proliferation markers such as Cyclin D1, proliferating cell nuclear antigen (PCNA) and an increase in NF- $\kappa$ B signalling [94]. In human hepatocellular carcinoma, SATB1 was shown to promote cell cycle progression and cell proliferation [59]. This occurred through upregulation of cyclin dependent kinase (CDK) 4 and inhibition of the tumour suppressor protein p16INK4A [59]. In cutaneous CD30+ lymphoproliferative disease, a common cutaneous T cell lymphoma, 
upregulated SATB1 was found to promote malignant cell proliferation through the repression of the CDK inhibitor $\mathrm{p} 21$, a negative mediator of cell cycle progression and an effector of a number of tumour suppressive pathways, thus inducing malignant cell proliferation $[95,96]$.

SATB1 was also shown to mediate epithelial to mesenchymal transition (EMT) [65]. EMT is the process by which immotile polarized, epithelial cells lose adherence at tight junctions, and become migratory mesenchymal cells [97]. As a key inducer of EMT, the transcription factor Snail, plays an important role in embryonic development and cancer progression by repressing the expression of E-cadherin [98]. SATB1 was shown to downregulate E-cadherin expression and upregulate inducers of EMT such as Snail1, Slug, Twist and vimentin in hepatocellular bladder and prostate cancers [59, 62, 65]. SATB1 also promoted drug-induced EMT in breast cancer cell lines, driven by the positive feedback regulation of miR-448 and NF- $\mathrm{BB}$ signalling [99]. Here, down-regulation of miR-448 increased SATB1 expression leading to upregulation of EGFR and progression into malignant phenotypes.

\section{SATB1 and apoptosis in cancer}

Apoptosis is the programmed cell death of damaged cells and is an important process in tissue homeostasis. Disruption of apoptosis is a cardinal feature in the development of cancer. BCL2 is a proto-oncogene and an anti-apoptotic protein that contributes to cancer pathogenesis by interrupting the cell death pathway and promoting the survival of damaged cells [100]. In several types of tumours, SATB1 has been shown to induce increased BCL2 expression, interfering with apoptosis in cancer cells [67, 70].

SATB1 regulates long-range transcription of BCL2 gene through the major breakpoint region (mbr) on the 3'untranslated region (UTR) of the BCL2 gene [101, 102]. SATB1 mediated chromatin looping facilitates the interaction between the mbr and BCL2 promoter resulting in increased BCL2 transcription. Likewise, SATB1 induced epigenetic modification 
of BCL2 promoter enables binding of the transcription factor CREB to BCL2 promoter and positively regulates BCL2 expression [103]. SATB1 also regulates the expression of the proapoptotic gene NOXA via the mbr enhancer [104]. Transcription of BCL2 and NOXA genes depends on their interaction with mbr enhancer. If the mbr enhancer binds to the BCL2 promoter, then the BCL2 gene is transcribed and NOXA is repressed and vice versa. [104]. In normal conditions, on apoptotic stimulation, SATB1 is cleaved by the protease Caspase6 reducing SATB1 induced BCL2 expression. Moreover, in Jurkat cells, reduced SATB1 levels were shown to mediate increased expression of NOXA, decreasing BCL2 expression. However, inhibition of SATB1 cleavage by caspase-6 inhibitor led to an increase in BCL2 expression and decrease in NOXA transcription [104]. Thus increase in SATB1 levels, may potentially disrupt the equilibrium of the apoptotic regulatory genes and may contribute to cancer development and progression.

Studies show that aberrant SATB1 expression is associated with abnormal BCL2 expression, apoptosis, and tumorigenesis $[67,70]$. Studies in glioma show that SATB1 expression was significantly high in approximately $63 \%$ of tumour samples analysed with the simultaneous increase in expression of the cell proliferation marker Ki67. These were associated with poor survival [70]. Further, shRNA mediated silencing of SATB1 expression in vivo decreased angiogenesis and altered expression of c-Met, SLC22A18, caspase-3 and BCL2 protein increasing apoptosis and reducing tumour growth and progression. In human glioblastoma multiforme (GBM), SATB1 mRNA expression was upregulated in samples with a concurrent increase in the levels of BCL2 and PCNA protein levels and showed decreased apoptosis compared with the normal brain tissues [67]. The increase in SATB1 expression and BCL2 levels were positively associated with reduced survival rates and poor prognosis [67]. Knocking down SATB1 expression, consequently increased caspase-3/-7 activity with a concurrent decrease in BCL2 and PCNA levels [67]. These studies implicate SATB1 in brain tumour progression mediated by its effects on reduced apoptosis and increased cell 
proliferation. Further, in human liver cancers, SATB1 was also shown to inhibit apoptosis by suppressing the FADD caspase-8 caspase-3-death receptor mediated apoptosis apoptotic pathway [59]. These studies indicate a functional role of SATB1 in apoptosis regulation, whereby aberrant expression of SATB1 supports cancer progression.

\section{SATB1 in invasion and metastasis}

In the aggressive breast cancer cell line MDA-MB-231, SATB1 was associated with the increased expression of EGF receptors (ERBB1, ERBB2, ERBB3, and ERBB4), and EGFR ligands, neuregulin (NRG) and amphiregulin (AREG). In addition, SATB1 was also shown to upregulate the expression of metastasis promoting genes; metastatin (S100A4), vascular endothelial growth factor B, MMP-2, 3 and 9, TGF- $\beta$ and connective tissue growth factor [1]. Whilst, the expression of metastasis suppressor genes; BRMS1, KAI1, KISS1, NME1 were was inhibited by SATB1 [1]. Other studies defined the role of SATB1 in invasion and metastasis. In hepatocellular carcinoma, SATBI promoted in vivo tumour growth and increased metastatic potential [59]. In colorectal cancer, overexpressed SATB1 increased the expression of the invasion marker MMP2, along with the loss of expression of tumour suppressor gene Adenomatous polyposis coli (APC) [94].

\section{SATB1 and multidrug resistance}

SATB1 is upregulated in multidrug-resistant (MDR) breast cancer lines [40]. Along with higher invasive potential, EMT and metastasis, SATB1 also conferred MDR to these cells via effects on P-glycoprotein (P-gp) expression [40]. Further, the MDR phenotype of these cells was partly reversed by the suppression of SATB1. SATB1 also contributes to MDR through non P-gp dependent mechanisms via the suppression of drug-induced apoptosis [40]. 
SATB1 has been shown to contribute to MDR in nasopharyngeal carcinoma, gastric cancer cell Hines and osteosarcoma cell lines $[48,52,105]$.

\section{Future directions}

SATB1 expression is associated with cancer progression, dissemination and resistance and is of prognostic significance. As SATB1 regulates many genes depending on cell type and context, it is important to elucidate the mechanism through which SATB1 mediates tumorigenesis and tumour progression in different cancers. SATB1 regulates several genes important in normal biological processes including the immune responses that are important in cancer survival, therefore further studies are required to interrogate the role of SATB1mediated immune responses in cancers.

Most studies have examined SATB1 gene expression and protein levels but fail to consider the effects of post-translational modifications of SATB1 mechanistically and its significance on cancer pathogenesis. Further, it is also important to identify approaches to differentiate SATB1 from its functionally distinct homologue SATB2. Thus, appropriate techniques to analyse SATB1 expression and the effects of its inhibition on downstream pathways are further required for it to be used as a potential target in cancer therapy.

In summary, SATB1 plays an important role in various cellular processes and biological pathways and its deregulation is implicated in cancer development and growth and has the potential to serve as a biomarker and a therapeutic target in cancer treatment. 


\section{References}

[1] H.J. Han, J. Russo, Y. Kohwi, T. Kohwi-Shigematsu, SATB1 reprogrammes gene expression to promote breast tumour growth and metastasis, Nature 452(7184) (2008) 187-93. [2] R. Mir, S.J. Pradhan, P. Patil, R. Mulherkar, S. Galande, Wnt/beta-catenin signaling regulated SATB1 promotes colorectal cancer tumorigenesis and progression, Oncogene 35(13) (2016) 1679-91.

[3] J. Ma, K. Wu, Z. Zhao, R. Miao, Z. Xu, Special AT-rich sequence binding protein 1 promotes tumor growth and metastasis of esophageal squamous cell carcinoma, Tumour biology : the journal of the International Society for Oncodevelopmental Biology and Medicine 39(3) (2017) 1010428317694537.

[4] R. Berezney, M.J. Mortillaro, H. Ma, X. Wei, J. Samarabandu, The nuclear matrix: a structural milieu for genomic function, International review of cytology 162a (1995) 1-65.

[5] L.A. Dickinson, T. Joh, Y. Kohwi, T. Kohwi-Shigematsu, A tissue-specific MAR/SAR DNA-binding protein with unusual binding site recognition, Cell 70(4) (1992) 631-45.

[6] I. de Belle, S. Cai, T. Kohwi-Shigematsu, The genomic sequences bound to special ATrich sequence-binding protein 1 (SATB1) in vivo in Jurkat T cells are tightly associated with the nuclear matrix at the bases of the chromatin loops, The Journal of cell biology 141(2) (1998) $335-48$.

[7] S. Cai, H.J. Han, T. Kohwi-Shigematsu, Tissue-specific nuclear architecture and gene expression regulated by SATB1, Nature genetics 34(1) (2003) 42-51.

[8] S. Cai, C.C. Lee, T. Kohwi-Shigematsu, SATB1 packages densely looped, transcriptionally active chromatin for coordinated expression of cytokine genes, Nature genetics 38(11) (2006) $1278-88$.

[9] D. Yasui, M. Miyano, S. Cai, P. Varga-Weisz, T. Kohwi-Shigematsu, SATB1 targets chromatin remodelling to regulate genes over long distances, Nature 419(6907) (2002) 641-5. 
[10] P. Pavan Kumar, P.K. Purbey, C.K. Sinha, D. Notani, A. Limaye, R.S. Jayani, S. Galande, Phosphorylation of SATB1, a global gene regulator, acts as a molecular switch regulating its transcriptional activity in vivo, Molecular cell 22(2) (2006) 231-43.

[11] P.K. Purbey, S. Singh, D. Notani, P.P. Kumar, A.S. Limaye, S. Galande, Acetylationdependent interaction of SATB1 and CtBP1 mediates transcriptional repression by SATB1, Molecular and cellular biology 29(5) (2009) 1321-37.

[12] P.K. Purbey, S. Singh, P.P. Kumar, S. Mehta, K.N. Ganesh, D. Mitra, S. Galande, PDZ domain-mediated dimerization and homeodomain-directed specificity are required for highaffinity DNA binding by SATB1, Nucleic acids research 36(7) (2008) 2107-22.

[13] D. Notani, A.S. Limaye, P.P. Kumar, S. Galande, Phosphorylation-dependent regulation of SATB1, the higher-order chromatin organizer and global gene regulator, Methods in molecular biology (Clifton, N.J.) 647 (2010) 317-35.

[14] P.S. Foster, M. Plank, A. Collison, H.L. Tay, G.E. Kaiko, J. Li, S.L. Johnston, P.M. Hansbro, R.K. Kumar, M. Yang, J. Mattes, The emerging role of microRNAs in regulating immune and inflammatory responses in the lung, Immunological reviews 253(1) (2013) 198215.

[15] A.M. Lena, M. Mancini, P. Rivetti di Val Cervo, G. Saintigny, C. Mahe, G. Melino, E. Candi, MicroRNA-191 triggers keratinocytes senescence by SATB1 and CDK6 downregulation, Biochemical and biophysical research communications 423(3) (2012) 509-14. [16] N. Nagpal, H.M. Ahmad, B. Molparia, R. Kulshreshtha, MicroRNA-191, an estrogenresponsive microRNA, functions as an oncogenic regulator in human breast cancer, Carcinogenesis 34(8) (2013) 1889-99.

[17] G. Wang, B. Li, Y. Fu, M. He, J. Wang, P. Shen, L. Bai, miR-23a suppresses proliferation of osteosarcoma cells by targeting SATB1, Tumour biology : the journal of the International Society for Oncodevelopmental Biology and Medicine 36(6) (2015) 4715-21. 
[18] T. Papagiannakopoulos, A. Shapiro, K.S. Kosik, MicroRNA-21 targets a network of key tumor-suppressive pathways in glioblastoma cells, Cancer research 68(19) (2008) 8164-72.

[19] X. Zhang, C. Wang, S. Shan, X. Liu, Z. Jiang, T. Ren, TLR4/ROS/miRNA-21 pathway underlies lipopolysaccharide instructed primary tumor outgrowth in lung cancer patients, Oncotarget (2016).

[20] Y. Gao, Q. Cai, Y. Huang, S. Li, H. Yang, L. Sun, K. Chen, Y. Wang, MicroRNA-21 as a potential diagnostic biomarker for breast cancer patients: a pooled analysis of individual studies, Oncotarget (2016).

[21] M.V. Iorio, R. Visone, G. Di Leva, V. Donati, F. Petrocca, P. Casalini, C. Taccioli, S. Volinia, C.G. Liu, H. Alder, G.A. Calin, S. Menard, C.M. Croce, MicroRNA signatures in human ovarian cancer, Cancer research 67(18) (2007) 8699-707.

[22] Y. Han, G.X. Xu, H. Lu, D.H. Yu, Y. Ren, L. Wang, X.H. Huang, W.J. Hou, Z.H. Wei, Y.P. Chen, Y.G. Cao, R. Zhang, Dysregulation of miRNA-21 and their potential as biomarkers for the diagnosis of cervical cancer, Int J Clin Exp Pathol 8(6) (2015) 7131-9.

[23] G. Xu, Y. Zhang, J. Wei, W. Jia, Z. Ge, Z. Zhang, X. Liu, MicroRNA-21 promotes hepatocellular carcinoma HepG2 cell proliferation through repression of mitogen-activated protein kinase-kinase 3, BMC cancer 13 (2013) 469.

[24] B. Will, T.O. Vogler, B. Bartholdy, F. Garrett-Bakelman, J. Mayer, L. Barreyro, A. Pandolfi, T.I. Todorova, U.C. Okoye-Okafor, R.F. Stanley, T.D. Bhagat, A. Verma, M.E. Figueroa, A. Melnick, M. Roth, U. Steidl, Satb1 regulates the self-renewal of hematopoietic stem cells by promoting quiescence and repressing differentiation commitment, Nature immunology 14(5) (2013) 437-45.

[25] Y. Satoh, T. Yokota, T. Sudo, M. Kondo, A. Lai, P.W. Kincade, T. Kouro, R. Iida, K. Kokame, T. Miyata, Y. Habuchi, K. Matsui, H. Tanaka, I. Matsumura, K. Oritani, T. KohwiShigematsu, Y. Kanakura, The Satb1 protein directs hematopoietic stem cell differentiation toward lymphoid lineages, Immunity 38(6) (2013) 1105-15. 
[26] J.D. Alvarez, D.H. Yasui, H. Niida, T. Joh, D.Y. Loh, T. Kohwi-Shigematsu, The MARbinding protein SATB1 orchestrates temporal and spatial expression of multiple genes during T-cell development, Genes \& development 14(5) (2000) 521-35.

[27] M. Kondo, Y. Tanaka, T. Kuwabara, T. Naito, T. Kohwi-Shigematsu, A. Watanabe, SATB1 Plays a Critical Role in Establishment of Immune Tolerance, Journal of immunology (Baltimore, Md. : 1950) 196(2) (2016) 563-72.

[28] K. Kakugawa, S. Kojo, H. Tanaka, W. Seo, T.A. Endo, Y. Kitagawa, S. Muroi, M. Tenno, N. Yasmin, Y. Kohwi, S. Sakaguchi, T. Kowhi-Shigematsu, I. Taniuchi, Essential Roles of SATB1 in Specifying T Lymphocyte Subsets, Cell reports 19(6) (2017) 1176-1188.

[29] D. Notani, K.P. Gottimukkala, R.S. Jayani, A.S. Limaye, M.V. Damle, S. Mehta, P.K. Purbey, J. Joseph, S. Galande, Global regulator SATB1 recruits beta-catenin and regulates $\mathrm{T}(\mathrm{H}) 2$ differentiation in Wnt-dependent manner, PLoS biology 8(1) (2010) e1000296.

[30] M. Beyer, Y. Thabet, R.U. Muller, T. Sadlon, S. Classen, K. Lahl, S. Basu, X. Zhou, S.L. Bailey-Bucktrout, W. Krebs, E.A. Schonfeld, J. Bottcher, T. Golovina, C.T. Mayer, A. Hofmann, D. Sommer, S. Debey-Pascher, E. Endl, A. Limmer, K.L. Hippen, B.R. Blazar, R. Balderas, T. Quast, A. Waha, G. Mayer, M. Famulok, P.A. Knolle, C. Wickenhauser, W. Kolanus, B. Schermer, J.A. Bluestone, S.C. Barry, T. Sparwasser, J.L. Riley, J.L. Schultze, Repression of the genome organizer SATB1 in regulatory T cells is required for suppressive function and inhibition of effector differentiation, Nature immunology 12(9) (2011) 898-907. [31] Y. Kitagawa, N. Ohkura, Y. Kidani, A. Vandenbon, K. Hirota, R. Kawakami, K. Yasuda, D. Motooka, S. Nakamura, M. Kondo, I. Taniuchi, T. Kohwi-Shigematsu, S. Sakaguchi, Guidance of regulatory T cell development by Satb1-dependent super-enhancer establishment, Nature immunology 18(2) (2017) 173-183.

[32] A.J. Tesone, M.R. Rutkowski, E. Brencicova, N. Svoronos, A. Perales-Puchalt, T.L. Stephen, M.J. Allegrezza, K.K. Payne, J.M. Nguyen, J. Wickramasinghe, J. Tchou, M.E. Borowsky, G.A. Rabinovich, A.V. Kossenkov, J.R. Conejo-Garcia, Satb1 Overexpression 
Drives Tumor-Promoting Activities in Cancer-Associated Dendritic Cells, Cell reports 14(7) (2016) 1774-1786.

[33] P.P. Kumar, O. Bischof, P.K. Purbey, D. Notani, H. Urlaub, A. Dejean, S. Galande, Functional interaction between PML and SATB1 regulates chromatin-loop architecture and transcription of the MHC class I locus, Nature cell biology 9(1) (2007) 45-56.

[34] M.Y. Fessing, A.N. Mardaryev, M.R. Gdula, A.A. Sharov, T.Y. Sharova, V. Rapisarda, K.B. Gordon, A.D. Smorodchenko, K. Poterlowicz, G. Ferone, Y. Kohwi, C. Missero, T. Kohwi-Shigematsu, V.A. Botchkarev, p63 regulates Satb1 to control tissue-specific chromatin remodeling during development of the epidermis, The Journal of cell biology 194(6) (2011) 825-39.

[35] M.A. Balamotis, N. Tamberg, Y.J. Woo, J. Li, B. Davy, T. Kohwi-Shigematsu, Y. Kohwi, Satb1 ablation alters temporal expression of immediate early genes and reduces dendritic spine density during postnatal brain development, Molecular and cellular biology 32(2) (2012) 33347.

[36] A.A. Mills, B. Zheng, X.J. Wang, H. Vogel, D.R. Roop, A. Bradley, p63 is a p53 homologue required for limb and epidermal morphogenesis, Nature 398(6729) (1999) 708-13. [37] M. Denaxa, M. Kalaitzidou, A. Garefalaki, A. Achimastou, R. Lasrado, T. Maes, V. Pachnis, Maturation-promoting activity of SATB1 in MGE-derived cortical interneurons, Cell reports 2(5) (2012) 1351-62.

[38] A. Fromberg, K. Engeland, A. Aigner, The Special AT-rich Sequence Binding Protein 1 (SATB1) and its role in solid tumors, Cancer letters 417 (2018) 96-111.

[39] M. Ding, J. Pan, Z. Guo, Q. Liu, C. Yang, L. Mao, SATB1 is a Novel Molecular Target for Cancer Therapy, Cancer investigation 36(1) (2018) 28-36.

[40] Q.Q. Li, Z.Q. Chen, J.D. Xu, X.X. Cao, Q. Chen, X.P. Liu, Z.D. Xu, Overexpression and involvement of special AT-rich sequence binding protein 1 in multidrug resistance in human breast carcinoma cells, Cancer science 101(1) (2010) 80-6. 
[41] X. Wang, X. Yu, Q. Wang, Y. Lu, H. Chen, Expression and clinical significance of SATB1 and TLR4 in breast cancer, Oncology letters 14(3) (2017) 3611-3615.

[42] N. Patani, W. Jiang, R. Mansel, R. Newbold, K. Mokbel, The mRNA expression of SATB1 and SATB2 in human breast cancer, Cancer cell international 9 (2009) 18.

[43] X.F. Fang, Z.B. Hou, X.Z. Dai, C. Chen, J. Ge, H. Shen, X.F. Li, L.K. Yu, Y. Yuan, Special AT-rich sequence-binding protein 1 promotes cell growth and metastasis in colorectal cancer, World journal of gastroenterology 19(15) (2013) 2331-9.

[44] Y. Zhang, X. Tian, H. Ji, X. Guan, W. Xu, B. Dong, M. Zhao, M. Wei, C. Ye, Y. Sun, X. Yuan, C. Yang, C. Hao, Expression of SATB1 promotes the growth and metastasis of colorectal cancer, PloS one 9(6) (2014) e100413.

[45] J.H. Lv, F. Wang, M.H. Shen, X. Wang, X.J. Zhou, SATB1 expression is correlated with beta-catenin associated epithelial-mesenchymal transition in colorectal cancer, Cancer biology \& therapy 17(3) (2016) 254-61.

[46] B. Huang, H. Zhou, S. Wang, X.P. Lang, X. Wang, Effect of silencing SATB1 on proliferation, invasion and apoptosis of A549 human lung adenocarcinoma cells, Oncology letters 12(5) (2016) 3818-3824.

[47] B. Huang, H. Zhou, X. Wang, Z. Liu, Silencing SATB1 with siRNA inhibits the proliferation and invasion of small cell lung cancer cells, Cancer cell international 13(1) (2013) 8.

[48] C.S. Ye, D.N. Zhou, Q.Q. Yang, Y.F. Deng, Silencing SATB1 influences cell invasion, migration, proliferation, and drug resistance in nasopharyngeal carcinoma, International journal of clinical and experimental pathology 7(3) (2014) 914-22.

[49] G. Song, K. Liu, X. Yang, B. Mu, J. Yang, L. He, X. Hu, Q. Li, Y. Zhao, X. Cai, G. Feng, SATB1 plays an oncogenic role in esophageal cancer by up-regulation of FN1 and PDGFRB, Oncotarget 8(11) (2017) 17771-17784. 
[50] S. Zhai, J. Xue, Z. Wang, L. Hu, High expression of special AT-rich sequence binding protein-1 predicts esophageal squamous cell carcinoma relapse and poor prognosis, Oncology letters 14(6) (2017) 7455-7460.

[51] C. Hedner, A. Gaber, D. Korkocic, B. Nodin, M. Uhlen, E. Kuteeva, H. Johannesson, K. Jirstrom, J. Eberhard, SATB1 is an independent prognostic factor in radically resected upper gastrointestinal tract adenocarcinoma, Virchows Archiv : an international journal of pathology 465(6) (2014) 649-59.

[52] F. Sun, X. Lu, H. Li, Z. Peng, K. Wu, G. Wang, Q. Tong, Special AT-rich sequence binding protein 1 regulates the multidrug resistance and invasion of human gastric cancer cells, Oncology letters 4(1) (2012) 156-162.

[53] Z. Chen, Z. Li, W. Li, Y. Zong, Y. Zhu, Y. Miao, Z. Xu, SATB1 Promotes Pancreatic Cancer Growth and Invasion Depending on MYC Activation, Digestive diseases and sciences 60(11) (2015) 3304-17.

[54] L. Guo, J. Zheng, T. Yu, Y. Liu, L. Duo, Elevated expression of SATB1 is involved in pancreatic tumorigenesis and is associated with poor patient survival, Molecular medicine reports 16(6) (2017) 8842-8848.

[55] J. Xiang, L. Zhou, S. Li, X. Xi, J. Zhang, Y. Wang, Y. Yang, X. Liu, X. Wan, AT-rich sequence binding protein 1: Contribution to tumor progression and metastasis of human ovarian carcinoma, Oncology letters 3(4) (2012) 865-870.

[56] B. Nodin, C. Hedner, M. Uhlen, K. Jirstrom, Expression of the global regulator SATB1 is an independent factor of poor prognosis in high grade epithelial ovarian cancer, Journal of ovarian research 5(1) (2012) 24.

[57] Y.C. Xu, C.J. Liang, D.X. Zhang, G.Q. Li, X. Gao, J.Z. Fu, F. Xia, J.J. Ji, L.J. Zhang, G.M. Li, J.X. Wu, LncSHRG promotes hepatocellular carcinoma progression by activating HES6, Oncotarget 8(41) (2017) 70630-70641. 
[58] D. Wu, L. Zeng, F. Liu, Q. Zhong, D. Zhang, C. Cai, W. Zhang, L. Wu, H. Chen, Special AT-rich DNA-binding protein-1 expression is associated with liver cancer metastasis, Oncology letters 12(6) (2016) 4377-4384.

[59] W. Tu, M. Luo, Z. Wang, W. Yan, Y. Xia, H. Deng, J. He, P. Han, D. Tian, Upregulation of SATB1 promotes tumor growth and metastasis in liver cancer, Liver international : official journal of the International Association for the Study of the Liver 32(7) (2012) 1064-78.

[60] L.J. Mao, J. Zhang, N. Liu, L. Fan, D.R. Yang, B.X. Xue, Y.X. Shan, J.N. Zheng, Oncolytic virus carrying shRNA targeting SATB1 inhibits prostate cancer growth and metastasis, Tumour biology : the journal of the International Society for Oncodevelopmental Biology and Medicine 36(11) (2015) 9073-81.

[61] L. Mao, C. Yang, J. Wang, W. Li, R. Wen, J. Chen, J. Zheng, SATB1 is overexpressed in metastatic prostate cancer and promotes prostate cancer cell growth and invasion, Journal of translational medicine 11 (2013) 111.

[62] H. Qi, X. Fu, Y. Li, X. Pang, S. Chen, X. Zhu, F. Li, W. Tan, SATB1 promotes epithelialmesenchymal transition and metastasis in prostate cancer, Oncology letters 13(4) (2017) 25772582.

[63] S. Shukla, H. Sharma, A. Abbas, G.T. MacLennan, P. Fu, D. Danielpour, S. Gupta, Upregulation of SATB1 is associated with prostate cancer aggressiveness and disease progression, PloS one 8(1) (2013) e53527.

[64] B. Han, L. Luan, Z. Xu, B. Wu, Expression and biological roles of SATB1 in human bladder cancer, Tumour biology : the journal of the International Society for Oncodevelopmental Biology and Medicine 34(5) (2013) 2943-9.

[65] F. Wan, C. Cheng, Z. Wang, X. Xiao, H. Zeng, S. Xing, X. Chen, J. Wang, S. Li, Y. Zhang, W. Xiang, Z. Zhu, C. Johnson, Z. Zhu, SATB1 overexpression regulates the development and progression in bladder cancer through EMT, PloS one 10(2) (2015) e0117518. 
[66] A. Fromberg, M. Rabe, H. Oppermann, F. Gaunitz, A. Aigner, Analysis of cellular and molecular antitumor effects upon inhibition of SATB1 in glioblastoma cells, BMC cancer 17(1) (2017) 3 .

[67] S.H. Chu, Y.B. Ma, D.F. Feng, Z.Q. Li, P.C. Jiang, Correlation between SATB1 and Bcl2 expression in human glioblastoma multiforme, Molecular medicine reports 7(1) (2013) 13943.

[68] S.H. Chu, Z.M. Zhou, D.F. Feng, Y.B. Ma, Inhibition of human glioma U251 cells growth in vitro and in vivo by hydroxyapatite nanoparticle-assisted delivery of short hairpin RNAs against SATB1, Molecular biology reports 41(2) (2014) 977-86.

[69] S.H. Chu, Y.B. Ma, D.F. Feng, H. Zhang, J.H. Qiu, Z.A. Zhu, Z.Q. Li, P.C. Jiang, Relationship between SATB1 expression and prognosis in astrocytoma, Journal of clinical neuroscience : official journal of the Neurosurgical Society of Australasia 20(4) (2013) 543-7. [70] S.H. Chu, Y.B. Ma, D.F. Feng, H. Zhang, Z.A. Zhu, Z.Q. Li, P.C. Jiang, Upregulation of SATB1 is associated with the development and progression of glioma, Journal of translational medicine 10 (2012) 149.

[71] Z. Pan, W. Jing, K. He, L. Zhang, X. Long, SATB1 is Correlated with Progression and Metastasis of Breast Cancers: A Meta-Analysis, Cellular physiology and biochemistry : international journal of experimental cellular physiology, biochemistry, and pharmacology 38(5) (2016) 1975-83.

[72] C.L. Yuan, L. Li, X. Zhou, H. Liz, L. Han, Expression of SATB1 and HER2 in gastric cancer and its clinical significance, European review for medical and pharmacological sciences 20(11) (2016) 2256-64.

[73] S. Wang, L. Wang, Y. Zhang, Y. Liu, F. Meng, J. Ma, P. Shang, Y. Gao, Q. Huang, X. Chen, Special AT-rich sequence-binding protein 1: a novel biomarker predicting cervical squamous cell carcinoma prognosis and lymph node metastasis, Japanese journal of clinical oncology 45(9) (2015) 812-8. 
[74] X. Lu, C. Cheng, S. Zhu, Y. Yang, L. Zheng, G. Wang, X. Shu, K. Wu, K. Liu, Q. Tong, SATB1 is an independent prognostic marker for gastric cancer in a Chinese population, Oncology reports 24(4) (2010) 981-7.

[75] J. Elebro, M. Heby, A. Gaber, B. Nodin, L. Jonsson, R. Fristedt, M. Uhlen, K. Jirstrom, J. Eberhard, Prognostic and treatment predictive significance of SATB1 and SATB2 expression in pancreatic and periampullary adenocarcinoma, Journal of translational medicine 12 (2014) 289.

[76] H. Chen, M. Takahara, J. Oba, L. Xie, T. Chiba, S. Takeuchi, Y. Tu, T. Nakahara, H. Uchi, Y. Moroi, M. Furue, Clinicopathologic and prognostic significance of SATB1 in cutaneous malignant melanoma, Journal of dermatological science 64(1) (2011) 39-44.

[77] E. Iorns, H.J. Hnatyszyn, P. Seo, J. Clarke, T. Ward, M. Lippman, The role of SATB1 in breast cancer pathogenesis, Journal of the National Cancer Institute 102(16) (2010) 1284-96.

[78] C.I. Selinger, W.A. Cooper, S. Al-Sohaily, D.N. Mladenova, L. Pangon, C.W. Kennedy, B.C. McCaughan, C. Stirzaker, M.R. Kohonen-Corish, Loss of special AT-rich binding protein 1 expression is a marker of poor survival in lung cancer, Journal of thoracic oncology : official publication of the International Association for the Study of Lung Cancer 6(7) (2011) 117989.

[79] D.R. FitzPatrick, I.M. Carr, L. McLaren, J.P. Leek, P. Wightman, K. Williamson, P. Gautier, N. McGill, C. Hayward, H. Firth, A.F. Markham, J.A. Fantes, D.T. Bonthron, Identification of SATB2 as the cleft palate gene on 2q32-q33, Human molecular genetics 12(19) (2003) 2491-501.

[80] T.R. Liu, L.H. Xu, A.K. Yang, Q. Zhong, M. Song, M.Z. Li, L.J. Hu, F.J. Chen, Z.D. Hu, P. Han, M.S. Zeng, Decreased expression of SATB2: a novel independent prognostic marker of worse outcome in laryngeal carcinoma patients, PloS one 7(7) (2012) e40704.

[81] S. Wang, J. Zhou, X.Y. Wang, J.M. Hao, J.Z. Chen, X.M. Zhang, H. Jin, L. Liu, Y.F. Zhang, J. Liu, Y.Q. Ding, J.M. Li, Down-regulated expression of SATB2 is associated with 
metastasis and poor prognosis in colorectal cancer, The Journal of pathology 219(1) (2009) $114-22$.

[82] M.A. Mansour, T. Hyodo, S. Ito, K. Kurita, T. Kokuryo, K. Uehara, M. Nagino, M. Takahashi, M. Hamaguchi, T. Senga, SATB2 suppresses the progression of colorectal cancer cells via inactivation of MEK5/ERK5 signaling, The FEBS journal 282(8) (2015) 1394-405. [83] M.A. Mansour, T. Hyodo, K.A. Akter, T. Kokuryo, K. Uehara, M. Nagino, T. Senga, SATB1 and SATB2 play opposing roles in c-Myc expression and progression of colorectal cancer, Oncotarget 7(4) (2016) 4993-5006.

[84] N. Meyer, L.Z. Penn, Reflecting on 25 years with MYC, Nature reviews. Cancer 8(12) (2008) 976-90.

[85] J. Galon, A. Costes, F. Sanchez-Cabo, A. Kirilovsky, B. Mlecnik, C. Lagorce-Pages, M. Tosolini, M. Camus, A. Berger, P. Wind, F. Zinzindohoue, P. Bruneval, P.H. Cugnenc, Z. Trajanoski, W.H. Fridman, F. Pages, Type, density, and location of immune cells within human colorectal tumors predict clinical outcome, Science (New York, N.Y.) 313(5795) (2006) 19604.

[86] S.M. Mahmoud, E.C. Paish, D.G. Powe, R.D. Macmillan, M.J. Grainge, A.H. Lee, I.O. Ellis, A.R. Green, Tumor-infiltrating CD8+ lymphocytes predict clinical outcome in breast cancer, Journal of clinical oncology : official journal of the American Society of Clinical Oncology 29(15) (2011) 1949-55.

[87] E. Sato, S.H. Olson, J. Ahn, B. Bundy, H. Nishikawa, F. Qian, A.A. Jungbluth, D. Frosina, S. Gnjatic, C. Ambrosone, J. Kepner, T. Odunsi, G. Ritter, S. Lele, Y.T. Chen, H. Ohtani, L.J. Old, K. Odunsi, Intraepithelial CD8+ tumor-infiltrating lymphocytes and a high $\mathrm{CD} 8+$ /regulatory $\mathrm{T}$ cell ratio are associated with favorable prognosis in ovarian cancer, Proceedings of the National Academy of Sciences of the United States of America 102(51) (2005) 18538-43. 
[88] C. Hermans, D. Anz, J. Engel, T. Kirchner, S. Endres, D. Mayr, Analysis of FoxP3+ Tregulatory cells and CD8+ T-cells in ovarian carcinoma: location and tumor infiltration patterns are key prognostic markers, PloS one 9(11) (2014) e111757.

[89] H. Harlin, T.V. Kuna, A.C. Peterson, Y. Meng, T.F. Gajewski, Tumor progression despite massive influx of activated CD8(+) $\mathrm{T}$ cells in a patient with malignant melanoma ascites, Cancer immunology, immunotherapy : CII 55(10) (2006) 1185-97.

[90] T.L. Stephen, K.K. Payne, R.A. Chaurio, M.J. Allegrezza, H. Zhu, J. Perez-Sanz, A. Perales-Puchalt, J.M. Nguyen, A.E. Vara-Ailor, E.B. Eruslanov, M.E. Borowsky, R. Zhang, T.M. Laufer, J.R. Conejo-Garcia, SATB1 Expression Governs Epigenetic Repression of PD-1 in Tumor-Reactive T Cells, Immunity 46(1) (2017) 51-64.

[91] J.R. Brahmer, S.S. Tykodi, L.Q. Chow, W.J. Hwu, S.L. Topalian, P. Hwu, C.G. Drake, L.H. Camacho, J. Kauh, K. Odunsi, H.C. Pitot, O. Hamid, S. Bhatia, R. Martins, K. Eaton, S. Chen, T.M. Salay, S. Alaparthy, J.F. Grosso, A.J. Korman, S.M. Parker, S. Agrawal, S.M. Goldberg, D.M. Pardoll, A. Gupta, J.M. Wigginton, Safety and activity of anti-PD-L1 antibody in patients with advanced cancer, The New England journal of medicine 366(26) (2012) 245565.

[92] W. Zou, J.D. Wolchok, L. Chen, PD-L1 (B7-H1) and PD-1 pathway blockade for cancer therapy: Mechanisms, response biomarkers, and combinations, Science translational medicine 8(328) (2016) 328rv4.

[93] J.R. Cubillos-Ruiz, J.R. Baird, A.J. Tesone, M.R. Rutkowski, U.K. Scarlett, A.L. Camposeco-Jacobs, J. Anadon-Arnillas, N.M. Harwood, M. Korc, S.N. Fiering, L.F. Sempere, J.R. Conejo-Garcia, Reprogramming tumor-associated dendritic cells in vivo using miRNA mimetics triggers protective immunity against ovarian cancer, Cancer research 72(7) (2012) 1683-93. 
[94] J. Zhang, B. Zhang, X. Zhang, Y. Sun, X. Wei, M.A. McNutt, S. Lu, Y. Liu, D. Zhang, M. Wang, Z. Lin, N. Niu, SATB1 expression is associated with biologic behavior in colorectal carcinoma in vitro and in vivo, PloS one 8(1) (2013) e47902.

[95] Y. Wang, X. Gu, G. Zhang, L. Wang, T. Wang, Y. Zhao, X. Zhang, Y. Zhou, M. Kadin, P. Tu, SATB1 overexpression promotes malignant T-cell proliferation in cutaneous CD30+ lymphoproliferative disease by repressing p21, Blood 123(22) (2014) 3452-61.

[96] T. Abbas, A. Dutta, p21 in cancer: intricate networks and multiple activities, Nature reviews. Cancer 9(6) (2009) 400-14.

[97] R. Kalluri, R.A. Weinberg, The basics of epithelial-mesenchymal transition, The Journal of clinical investigation 119(6) (2009) 1420-8.

[98] Y. Wang, J. Shi, K. Chai, X. Ying, B.P. Zhou, The Role of Snail in EMT and Tumorigenesis, Current cancer drug targets 13(9) (2013) 963-972.

[99] Q.Q. Li, Z.Q. Chen, X.X. Cao, J.D. Xu, J.W. Xu, Y.Y. Chen, W.J. Wang, Q. Chen, F. Tang, X.P. Liu, Z.D. Xu, Involvement of NF-kappaB/miR-448 regulatory feedback loop in chemotherapy-induced epithelial-mesenchymal transition of breast cancer cells, Cell death and differentiation 18(1) (2011) 16-25.

[100] S. Cory, J.M. Adams, The Bcl2 family: regulators of the cellular life-or-death switch, Nature reviews. Cancer 2(9) (2002) 647-56.

[101] J. Zhang, C. Ma, X. Han, L.K. Durrin, Y. Sun, The bcl-2 major breakpoint region (mbr) possesses transcriptional regulatory function, Gene 379 (2006) 127-31.

[102] C. Ma, J. Zhang, L.K. Durrin, J. Lv, D. Zhu, X. Han, Y. Sun, The BCL2 major breakpoint region (mbr) regulates gene expression, Oncogene 26(18) (2007) 2649-57.

[103] F. Gong, L. Sun, Z. Wang, J. Shi, W. Li, S. Wang, X. Han, Y. Sun, The BCL2 gene is regulated by a special AT-rich sequence binding protein 1-mediated long range chromosomal interaction between the promoter and the distal element located within the 3'-UTR, Nucleic acids research 39(11) (2011) 4640-52. 
[104] Y. Yang, Z. Wang, L. Sun, L. Shao, N. Yang, D. Yu, X. Zhang, X. Han, Y. Sun, SATB1 Mediates Long-Range Chromatin Interactions: A Dual Regulator of Anti-Apoptotic BCL2 and Pro-Apoptotic NOXA Genes, PloS one 10(9) (2015) e0139170.

[105] H. Zhang, X. Su, L. Guo, L. Zhong, W. Li, Z. Yue, X. Wang, Y. Mu, X. Li, R. Li, Z. Wang, Silencing SATB1 inhibits the malignant phenotype and increases sensitivity of human osteosarcoma U2OS cells to arsenic trioxide, International journal of medical sciences 11(12) (2014) 1262-9.

[106] T. Yokota, Y. Kanakura, Role of tissue-specific AT-rich DNA sequence-binding proteins in lymphocyte differentiation, International journal of hematology 100(3) (2014) 238-45. 


\section{Figure Legends:}

Figure 1. SATB1 mediated chromatin loop formation. SATB1 forms chromatin loops through the anchoring of chromatin to the nuclear matrix and mediates long distance gene regulation providing docking sites for histone remodelling enzymes and transcription factors. Figure adapted from [106]

Figure 2. Primary protein structure of SATB1 with its functional domains.

The nuclear localization signal domain (NLS), the protein binding domain PDZ, the Base Unpairing Region (BURs) and the DNA binding region, the homeodomain (HD). [Figure adapted from http://atlasgeneticsoncology.org/Genes/SATB1ID44225ch3p24.html]

Figure 3. Depicts post-translational modifications of SATB1-induced gene regulation. Phosphorylation of SATB1 leads to its interaction with histone deacetylase 1 (HDAC1) and CtBP1 resulting in repression of gene expression [10]. On de-phosphorylation, SATB1 is acetylated as it interacts with acetyltransferase PCAF, leading to the disassociation of HDAC1 and CtBP1 and activation of gene expression [11].

Figure 4. Role of SATB1 in cancer. It plays important roles in regulating the tumour microenvironment, SATB1 upregulates the expression of proliferation markers such as proliferating cell nuclear antigen (PCNA) and Cyclin D1 and also increases the expression of proto-oncogene MYC. Altered SATB1 expression implicates CD+8 T cells and DCs in impaired tumour immunity. SATB1 also alters the expression of epithelial-mesenchymal transition (EMT) markers, E-cadherin, Snail 1 and Slug and thus promotes cancer. Overexpression of SATB1 induces increases in anti-apoptotic BCL2 expression and decreases the activity of Caspases which results in suppression of apoptosis in cancers. SATB1 promotes 
invasion through the up-regulation of epidermal growth factor (EGF) receptors and also stimulates the metastatic genes such as Metastatin and tumour growth factor (TGF)- $\beta$. In cancers, SATB1 also confers MDR through both P-gp and non P-gp mechanisms. 